\title{
Novel compound heterozygous nonsense variants, p.L150* and p.Y3565*, of the $U S H 2 A$ gene in a Chinese pedigree are associated with Usher syndrome type IIA
}

\author{
JIEWEN FU $^{1 *}$, JINGLIANG CHENG ${ }^{1 *}$, QI ZHOU ${ }^{2}$, MD. ASADUZZAMAN KHAN $^{1}$, \\ CHENGXIA DUAN ${ }^{2}$, JIANGZHOU PENG ${ }^{3}$, HONGBIN LV ${ }^{2}$ and JUNJIANG FU ${ }^{1}$ \\ ${ }^{1}$ Key Laboratory of Epigenetics and Oncology, Research Center for Preclinical Medicine, \\ Southwest Medical University; ${ }^{2}$ Department of Ophthalmology, The Affiliated Hospital of Southwest Medical University, \\ Luzhou, Sichuan $646000 ;{ }^{3}$ Department of Thoracic Surgery, The Third Affiliated Hospital \\ of Southern Medical University, Guangzhou, Guangdong 510630, P.R. China
}

Received January 8, 2020; Accepted July 6, 2020

DOI: $10.3892 / \mathrm{mmr} .2020 .11400$

\begin{abstract}
Usher syndrome refers to a group of genetically and clinically heterogeneous autosomal recessive diseases with retinitis pigmentosa (RP) and hearing deficiencies. The association between Usher syndrome-causative genes and resultant Usher syndrome phenotypes in patients are highly variable. In the present study, a Chinese family with Usher syndrome was recruited, and targeted next-generation sequencing, Sanger sequencing and segregation analysis were performed. The expression profiles and functional effects of the pathogenic variants of $U S H 2 A$ identified were analyzed. Novel nonsense compound heterozygous variants, c.T449G (p.L150*) and c.T10695A (p.Y3565*), were identified in the USH2A gene, which showed co-segregation with the disease phenotype causing Usher syndrome type IIA in the recruited Chinese pedigree. The p.L150* variant was predicted to produce a truncated protein which lacked almost all the functional domains of USH2A, whereas the p.Y3565* variant is located in one of the fibronectin type 3 domains, resulting in the loss of several fibronectin type 3 domains at the C-terminus of USH2A by producing the truncated protein. It was shown that $U s h 2 a$
\end{abstract}

Correspondence to: $\mathrm{Dr}$ Junjiang $\mathrm{Fu}$, Key Laboratory of Epigenetics and Oncology, Research Center for Preclinical Medicine, Southwest Medical University, 3-319 Zhongshan Road, Luzhou, Sichuan 646000, P.R. China

E-mail: fujunjiang@hotmail.com; fujunjiang@swmu.edu.cn

Dr Hongbin Lv, Department of Ophthalmology, The Affiliated Hospital of Southwest Medical University, 25 Taiping Street, Luzhou, Sichuan 646000, P.R. China

E-mail: oculistlvhongbin@163.com

${ }^{*}$ Contributed equally

Key words: Usher syndrome type IIA, usherin, truncated protein, targeted next-generation sequencing, genotype/phenotype correlation
mRNA expression levels were higher in the retina compared with those in the eye tissues (lens, sclera and cornea), uterus, ovary, breast, testis, spleen, kidney, liver, intestine, brain, skeletal muscle and blood. Additionally, the protein structure was shown to be highly conserved by comparing Homo sapiens USH2A to eight other species. To the best of our knowledge, the present study is the first to identify two novel pathogenic variants, c.T449G (p.L150*) and c.T10695A (p.Y3565*), in the $U S H 2 A$ gene in a patient with Usher syndrome type IIA, thereby expanding the known spectrums of USH2A causative mutations. The present discovery may assist in understanding the molecular pathogenesis underlying the development of RP and Usher syndrome type IIA, and in the development of diagnostic, therapeutic and genetic counseling strategies in patients with Usher syndrome type IIA disease.

\section{Introduction}

Usher syndrome is a group of genetically and clinically heterogeneous autosomal recessive diseases with progressive retinitis pigmentosa (RP) and sensorineural hearing deficiencies, including Usher syndrome types I, II and III (1-4). Patients with Usher syndrome type II (USH2) present with mild hearing impairments but normal vestibular responses, and type II is the most common type amongst the three types (1). Usher syndrome type II is genetically heterogeneous, including USH2A, USH2C and USH2D. Usher syndrome type IIA (USH2A locus) (OMIM, 276901) is the result of mutations of the USH2A gene (OMIM, 608400) (5), whereas USH2C (OMIM, 605472) is the result of mutations of the ADGRVI gene (OMIM, 602851) $(1,6)$ or by digenic mutations of both ADGRV1 and PDZD7 genes (OMIM, 612971) (7) and USH2D (OMIM, 611383) can be caused by mutations of the whirlin (WHRN) gene (OMIM, 607928) (8). As Hmani-Aifa et al (9) identified mutations of the adhesion G-protein coupled receptor V1 gene in an Usher II syndrome pedigree which had been previously mapped to the USH2B locus of chromosome 3p23-p24.2, the designation for the USH2B locus was withdrawn. 
Aliases for the USH2A gene include Usherin, USH2, US2, Usher Syndrome 2A, Usher Syndrome Type-2A Protein, Usher Syndrome Type IIa Protein, DJ1111A8.1 and RP39. This gene maps to chromosome 1q41, encoding a protein 5,202 amino acids in length that is comprised of a pentaxin motif, laminin epidermal growth factor (EGF) motifs and numerous fibronectin type III domains (10). Different isoforms have also been identified $(5,10-12)$. The protein is localized in the basement membrane and serves a vital role in the development and homeostasis of the inner ear and retina (2). Mutations of the USH2A gene have been identified in patients with Usher syndrome type IIA and non-syndromic RP (13). Eudy et al (5) was the first to identify $U S H 2 A$ mutations among patients with Usher syndrome type IIA.

The association between the variants in the Usher syndrome-causative genes and the resultant Usher syndrome phenotypes in patients are highly variable $(1,3,14)$. To the best of our knowledge, novel USH2A mutations in patients with Usher syndrome type IIA and association between the genotypes and phenotypes have not been well documented in the Chinese population. In the present study, by using targeted next-generation sequencing (TGS), Sanger sequencing and segregation analysis, novel nonsense, compound heterozygous mutations in the USH2A gene were identified in a Chinese pedigree with Usher syndrome.

\section{Materials and methods}

Pedigree and clinical assessment, blood collection, and DNA isolation. The M147 pedigree consisted of a proband and four family members from Sichuan, China (Fig. 1A). The proband was a 35-year-old female, and her parents, husband and son were 71, 60, 36 and 5-years-old, respectively. The blood samples of the proband were collected in June 2013, and TGS analysis was performed in December 2016. The blood samples of the four family members were collected in March 2019 for segregation analysis in our laboratory. For detailed clinical assessments, clinical history and ophthalmic examinations were performed with the proband, including fundoscopy, fundus photographs and fundus fluorescent photographs as previously described (15). Audiometric testing in the proband was also performed using pure-tone audiometry with different frequencies $(0.25,0.5,1,2,4$ and $8 \mathrm{kHz})(1)$. Written informed consent from the participants or guardians was obtained, and the study conformed to the Declaration of Helsinki. Blood samples were taken, and DNA was isolated from the family members $(16,17)$. DNA from blood samples was also taken from 200 healthy controls with an average age of 40.6 years-old (age ranges: 20-66; sex distribution: 2:3 female: Male) from Luzhou city between December 2016 and February 2017 without hearing and vision problems. The DNA integrity was verified by running the DNA sample on a $0.8 \%$ agarose gel. Written informed consent was obtained from the patients and healthy controls.

TGS. TGS analysis was performed on the proband M147 DNA, according to the manufacturer's protocol (Illumina, Inc.) as described previously (18-20). Library construction, TGS and data analysis were performed according to the manufacturer's protocols and as described previously $(19,21)$.

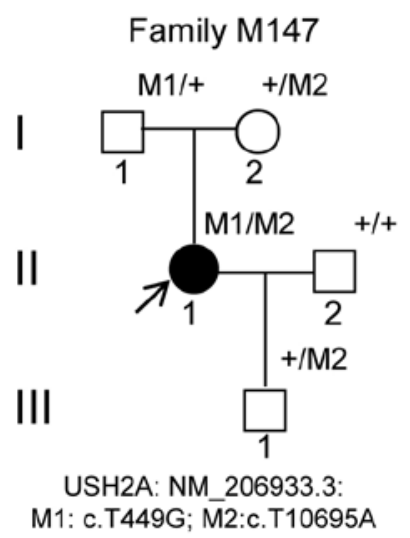

Figure 1. An M147 pedigree with Usher syndrome type IIA. Normal individuals are shown as a clear circle (female) or squares (males). The filled circle indicates the proband (II: 1, arrow) with the compound heterozygous mutation of the USH2A gene: NM_206933.3: c.T449G, c.T10695A. USH2A, usherin.

Sanger validation and segregation analysis. PCR amplification was performed for mutation validation. The primer pairs M147-USH-T10695A and M147-M201-USH were designed using the Primer3 program (http://bioinfo.ut.ee/primer3-0.4.0/) with genomic DNA sequences containing the NM_206933.3: c.T449G and c.T10695A, respectively, of the USH2A gene (Table I). PCR amplification was performed using the aforementioned primer pairs and the amplified PCR products were then sequenced using the Sanger method on an ABI-3500DX sequencer (Thermo Fisher Scientific, Inc.) (22) using M147-USH-T10695A-L and M147-M201-USH-L primers (Table I). Control samples from unrelated, ethnically matched individuals were also amplified using the primer pairs M147-USH-T10695A and M147-M201-USH and sequenced using the primers M147-USH-T10695A-L and M147-M201-USH-L. Segregation analysis in the M147 family was performed based on the sequencing results.

Prediction of protein structure and bioinformatics analysis. The homologs of the USH2A gene (NM_206933.3) in humans were analyzed using HomoloGene (ncbi.nlm.nih.gov/homolog ene $? \mathrm{Db}=$ homologene $\&$ Cmd=Retrieve \&list_uids $=66151$ ).

Conserved domains of the protein structure of USH2A (NP_996816.2) were searched using the NCBI conserved domain database (ncbi.nlm.nih.gov/Structure/cdd/wrpsb. cgi) (23-25).

$R N A$-sequencing (RNA-seq) profile, $R N A$ isolation and reverse transcription- $P C R(R T-P C R)$. To determine tissue specificity in humans, USH2A gene expression profiles were analyzed using RNA-seq data which was performed on normal samples from 95 human individuals representing 27 different tissues using: https://www.ncbi.nlm.nih.gov/gene/7399/?report=expression. The above RNA-seq data were collected from a project called HPA RNA-seq normal tissues (BioProject no. PRJEB4337).

Total RNA was extracted from tissues in mice using TRIzol $^{\circledR}$ reagent (Invitrogen; Thermo Fisher Scientific, Inc.). Experiments involving mice followed the international, national and institutional guidelines for the care and use of laboratory animals $(26,27,28)$ and was reviewed and approved 
Table I. Sequences of USH2A PCR primers.

\begin{tabular}{llcc}
\hline Primer name & \multicolumn{1}{c}{ Sequence, 5'-3' } & Size, bp & Mutation site \\
\hline M147-USH-T10695A & $\begin{array}{l}\text { F: AGGAACTGCTTGAGACAGCAA } \\
\text { R: CTGAACCCCTTTTCCCAGAG }\end{array}$ & 346 & c.T10695A \\
F147-M201-USH & $\begin{array}{l}\text { F: AGGCCTCAGTAGCTGCATCA } \\
\text { R: TTGGGGAAACAACTGGAAGA }\end{array}$ & 321 & c.T449G \\
FT- CGCTCTGCCTCTCCTCTCTA & 320 & N/A \\
RT-actin-m & $\begin{array}{l}\text { R: TTTATTGGAGGCTGCAAACC } \\
\text { F: TGTACCAACTGGGACGACA } \\
\text { R: TCTCAGCTGTGGTGGTGAAG }\end{array}$ & 392 & N/A
\end{tabular}

F, forward; R, reverse; N/A, not available. RT, reverse transcription.
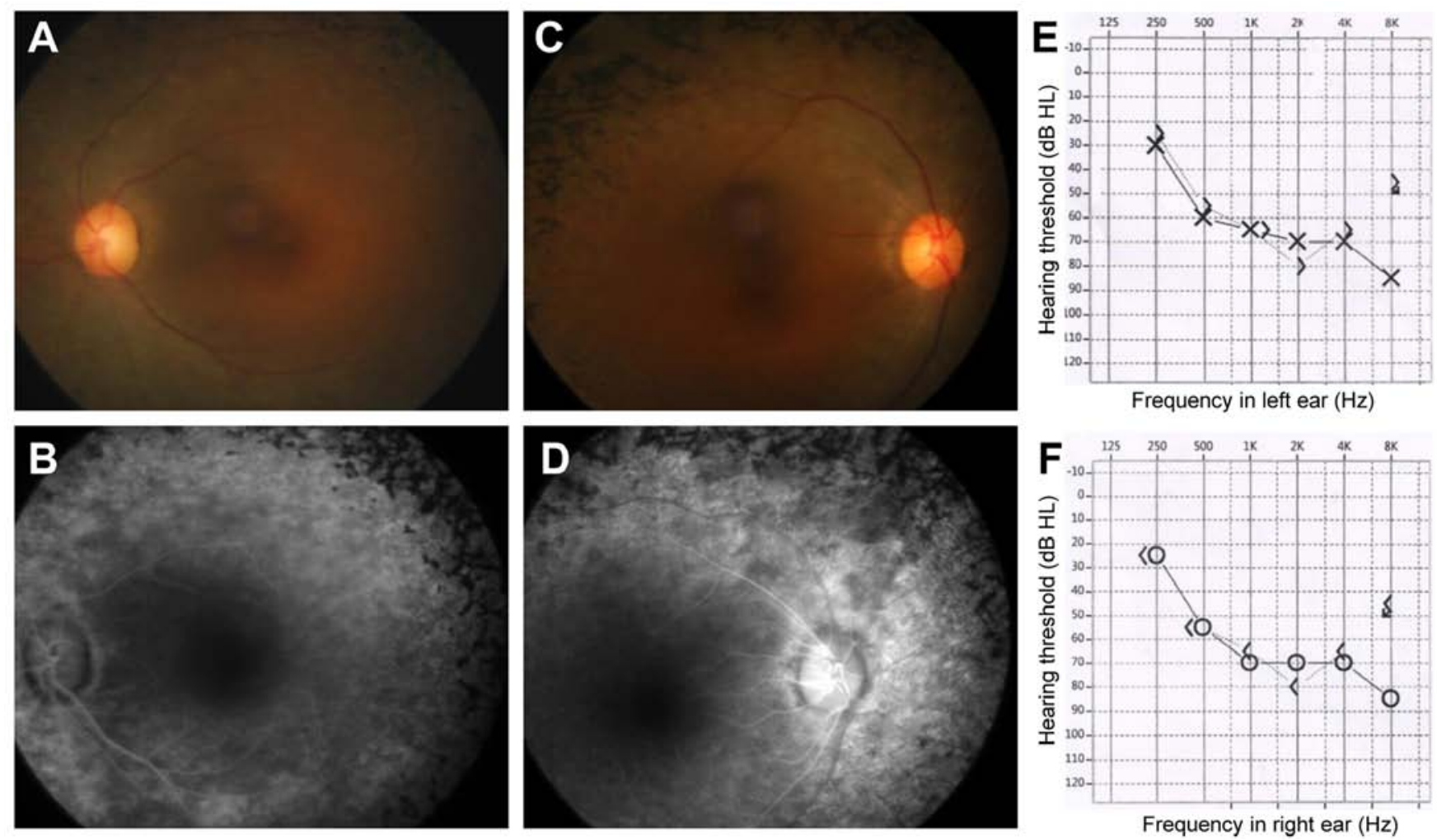

Figure 2. Representative retinal phenotypes and ear audiograms of proband II: 1. (A-D) Representative fundus photographs and fundus fluorescent photographs in patient II: 1 of both eyes. (E and F) Physiological audiograms of both ears of proband II:1.

by the Ethics Committee of Southwest Medical University, Sichuan, China. Male and female BALB/c mice (8 weeks old, $\sim 21 \mathrm{~g}$ ), were purchased from SPF (Beijing) Biotechnology Co., Ltd, China, and housed individually at room temperatures $\left(18-22^{\circ} \mathrm{C}\right)$ with $40-60 \%$ and with a $12-\mathrm{h}$ light/dark cycle. All mice were negative for pathogens. Mice received food and water ad libitum. At least five healthy mice for eye tissues and one healthy mouse for other tissues were used for RNA sampling. Retinal tissue at developmental stages, including whole embryo eye at 12.5 and 20.5 days before birth, and 2 weeks, 1 month, 2 and 3 months after birth, were used. Pentobarbital sodium $(200 \mathrm{mg} / \mathrm{kg}$ of body weight $)$ was intraperitoneally injected to mice for euthanasia. Death was verified as absence of vital signs, including no heartbeat, dilated pupils or cervical dislocation after anesthesia. First strand cDNA was then synthesized from $1 \mu \mathrm{g}$ total RNA with the ReverTra Ace ${ }^{\circledR}$ qPCR RT kit (cat. no. FSQ-201; Toyobo Life Science) according to the manufacturer's instructions and semi-quantitative RT-PCR was performed as described in our previous study $(29,30)$. Briefly, the PCR reaction system consisted of $5 \mu \mathrm{l} 2 \mathrm{X}$ Taq PCR MasterMix (Tiangen Biotech Co.), $1 \mu \mathrm{l} 2.5 \mu \mathrm{M}$ RT primers, $1 \mu \mathrm{l}$ RT products and $3 \mu \mathrm{l}$ double distilled $\mathrm{H}_{2} \mathrm{O}$. Amplification reactions were performed using the Applied Biosystems ${ }^{\circledR}$ Veriti ${ }^{\circledR}$ 96-Well Thermal Cycler PCR machine (Thermo Fisher Scientific, Inc.) with the following steps: Initial denaturation at $95^{\circ} \mathrm{C}$ for $90 \mathrm{sec}, 28-30$ cycles 
Table II. Characteristics of $U S H 2 A$ variants and disease-causing effects in the proband.

\begin{tabular}{lcllccc}
\hline \multirow{2}{*}{ Gene } & & \multicolumn{3}{c}{ Variation } & ExAC status \\
\cline { 3 - 5 } & Exon & Nucleotide & Protein & Type of mutation & Status & Ex \\
\hline USH2A & 2 & c.T449G & p.L150* & Nonsense & Heterozygous & Novel \\
USH2A & 54 & c.T10695A & p.Y3565* & Nonsense & Heterozygous & Novel \\
\hline
\end{tabular}

USH2A, usherin; c, variant at the cDNA level; p, variant at the protein level; ExAC, Exome Aggregation Consortium; *, stop codon.
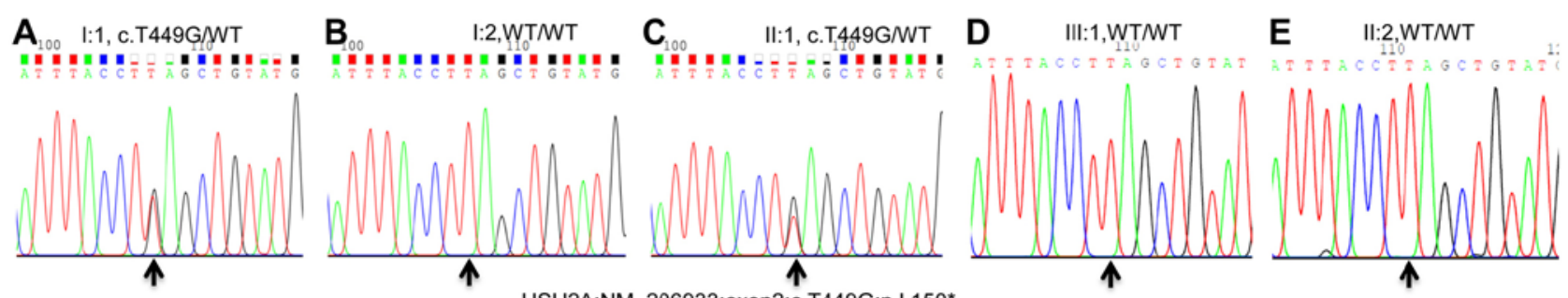

USH2A:NM_206933:exon2:c.T449G:p.L150*
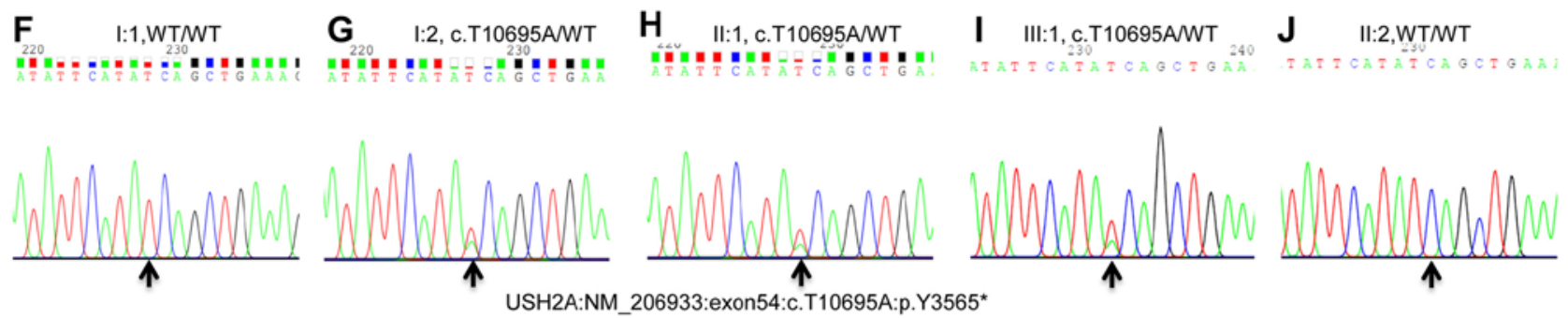

Figure 3. Pyrogram profiles for variant verification using Sanger sequencing. (A-E) Sequencing results pf I: 1 (heterozygous mutant type), I: 2 (WT), II: 1 (heterozygous mutant type), III: 1 (WT), and II: 2 (WT) of variant c.T449G. (F-J) Sequencing results of I: 1 (WT), I: 2 (heterozygous mutant type), II: 1 (heterozygous mutant type), III: 1 (heterozygous), and II: 2 (WT) of variant c.T10695A. The arrows indicate the mutation position of NM_206933.3: c.T449G or c.T10695A in the USH2A gene. USH2A, usherin; WT, wild-type.

of denaturation at $94^{\circ} \mathrm{C}$ for $30 \mathrm{sec}$, annealing at $72^{\circ} \mathrm{C}$ for $30 \mathrm{sec}$, extension at $72^{\circ} \mathrm{C}$ for $30 \mathrm{sec}$ and a final extension step at $72^{\circ} \mathrm{C}$ for $5 \mathrm{~min}$. Primer pairs for RT-ush $2 \mathrm{a}$ (RT-ush2a-L and RT-ush2a-R) targeting the mouse Ush $2 a$ gene, and the mouse $\beta$-actin gene which served as a control, are listed in Table I. RT-PCR products were separated on a $1.2 \%$ agarose gel in triplicate, and the gels were visualized by $0.5 \mu \mathrm{g} / \mathrm{ml}$ ethidium bromide (EB) staining. Densitometry was performed using ChemiDoc XR (version 5.2, Bio-Rad Laboratories, Inc.) $(17,31)$.

\section{Results}

Probandand clinical characteristics. The patient(Fig. 1A,II: 1) was a 35-year-old Chinese female. She suffered from night blindness since adolescence at age 12 and was first diagnosed with RP at the Yibin local county hospital 7 years ago. Fundus examinations showed bony spicule pigmentation and attenuated retinal vessels in both eyes, characteristic of a typical RP phenotype (Fig. 2A-D). The retinal pigment epitheliums were atrophied, and electroretinography results displayed no amplitude reactions (data not shown). The proband had a right naked eye vision of 0.6 and a left naked eye vision of 0.7 , and a visual acuity with correction of 0.8 in the right eye and $0.8+$ in the left eye. The logMAR or Snellen measurements were not available for this patient. The proband did not claim to exhibit hearing loss. The proband was characterized as RP. Based on genetic diagnostic results, pure tone audiometry testing was performed and found $65 \mathrm{~dB}$ hearing losses of both ears, indicating a moderate-to-severe binaural sensorineural hearing loss across all frequencies (Fig. 2E, left ear; Fig. 2F, right ear). Both the proband's parents and her son was normal. Thus, the proband was diagnosed with possible Usher syndrome type IIA.

Results of TGS and co-segregation analysis. Compound heterozygous mutations (c.T449G) in exon 2 and (c.T10695A) in exon 54 in the USH2A gene (NM_206933.3) were identified, leading to the presence of early stop codons (from TTA to TGA and from TAT to TAA, respectively) at amino acid positions 150 (p.L150*) and 3565 (p.Y3565*), respectively, in the USH2A protein (NP_996816.2; Fig. 1A, II: 1). Thus, both USH2A variants (c.T449G, p.L150*) and (c.T10695A, p.Y3565*) resulted in the production of truncated proteins, which were hypothesized to affect protein function. The characteristics of the $U S H 2 A$ variants and disease-causing effects of the proband are shown in Table II. Hence, these nonsense mutations (c.T449G, p.L150*) and (c.T10695A, p.Y3565*) in the USH2A gene affected protein function and supported the diagnosis of Usher syndrome type IIA. Both variants were revealed to be novel by 
A USH2A, H.sapiens

Usher syndrome $2 \mathrm{~A}$ (autosomal recessive, mild) USH2A, P.troglodytes

Usher syndrome 2A (autosomal recessive, mild) USH2A, M.mulatta

Usher syndrome $2 \mathrm{~A}$ (autosomal recessive, mild) USH2A, C.lupus

Usher syndrome $2 \mathrm{~A}$ (autosomal recessive, mild) USH2A, B.taurus

Usher syndrome $2 \mathrm{~A}$ (autosomal recessive, mild) Ush2a, M.musculus

Usher syndrome $2 \mathrm{~A}$ (autosomal recessive, mild) Ush2a, R.norvegicus

Usher syndrome $2 \mathrm{~A}$ (autosomal recessive, mild) USH2A, G.gallus

Usher syndrome $2 \mathrm{~A}$ (autosomal recessive, mild) ush2a, D.rerio

Usher syndrome $2 \mathrm{~A}$ (autosomal recessive, mild)
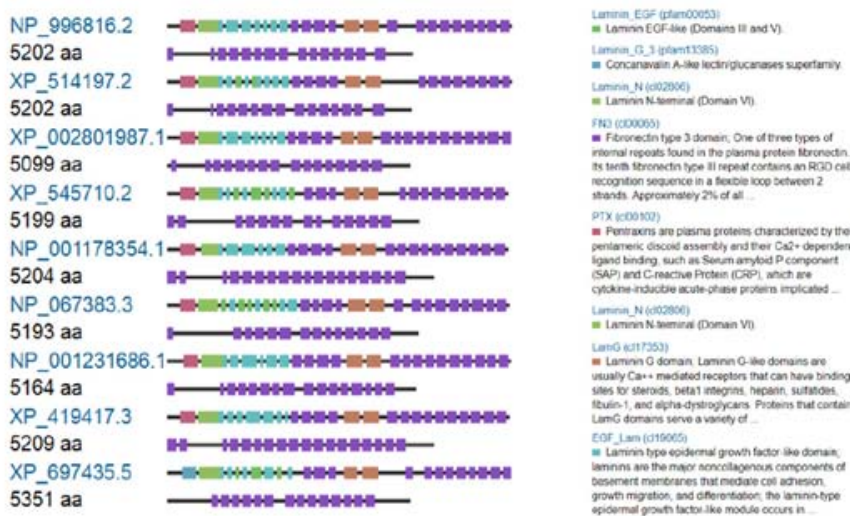

5351 aa

B

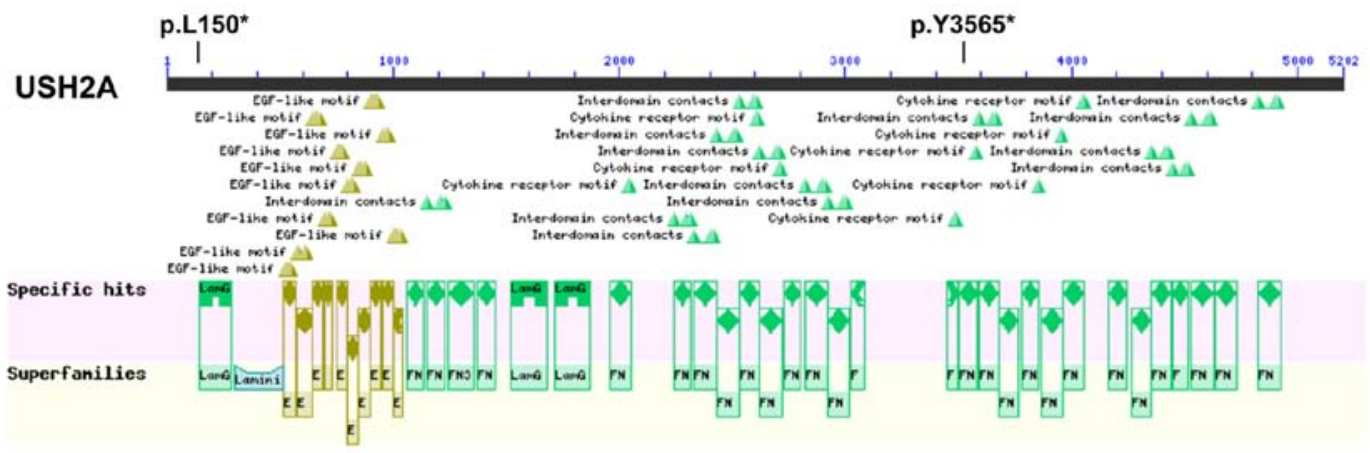

Figure 4. USH2A comparison, structure and mutant locations between different species. (A) Conservation analysis of USH2A in the indicated species (B) USH2A domains and mutant positions. Variants p.L150* and p.Y3565* of USH2A are indicated panel B. USH2A, usherin; aa, amino acids. EGF, epidermal growth factor; FN, fibronectin.

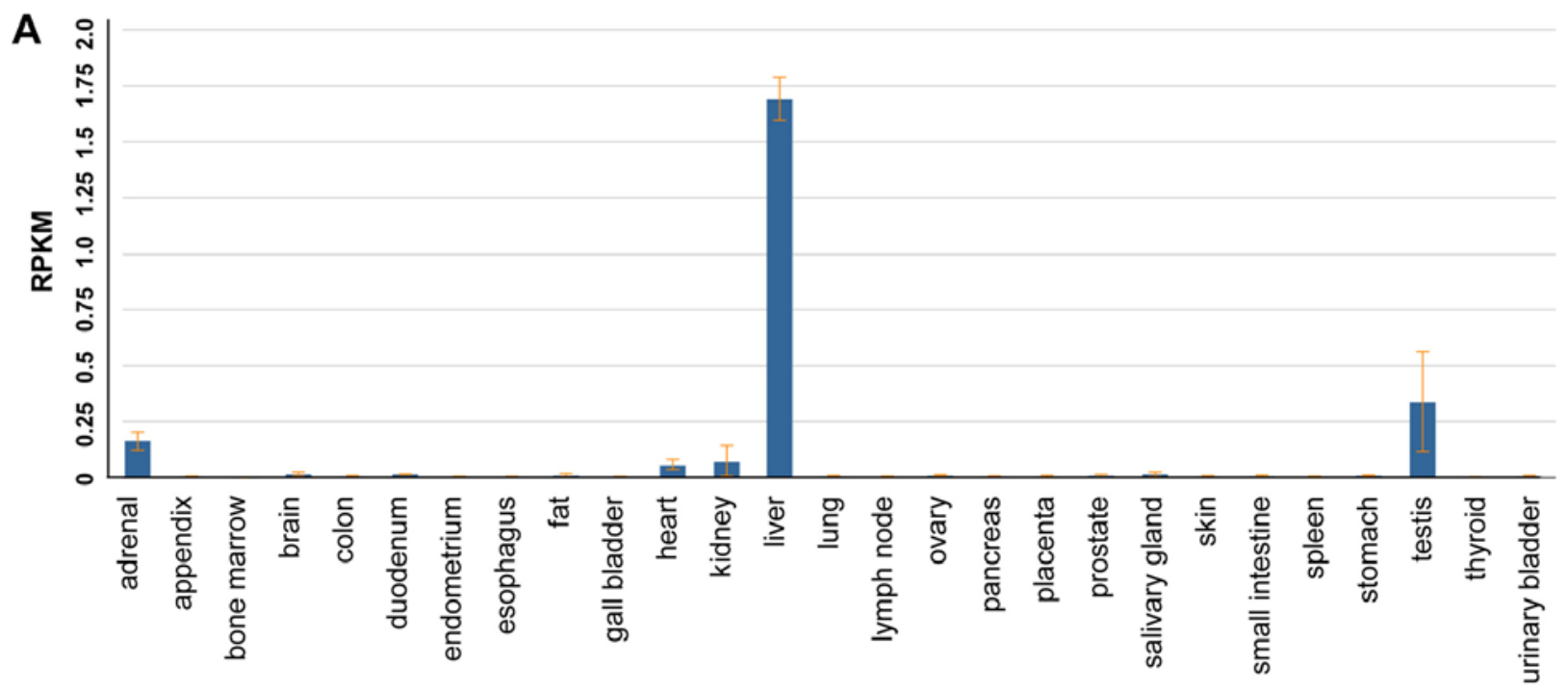

B

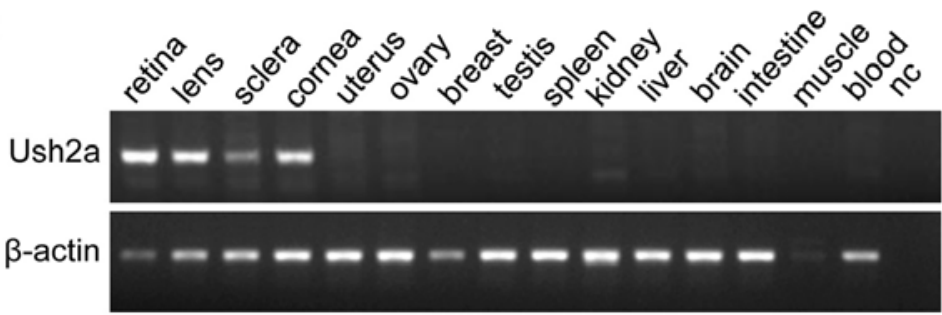

C

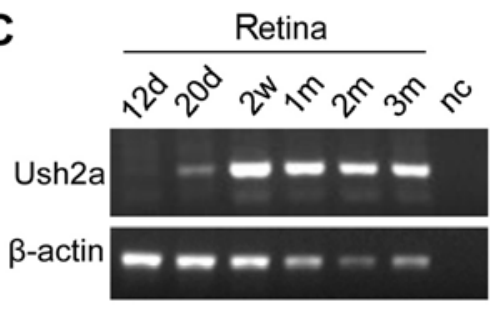

Figure 5. mRNA expression of USH2A in human tissues and Ush2a in mouse tissues. (A) USH2A mRNA expression in human tissues. The source of the data was derived from the link: https://www.ncbi.nlm.nih.gov/gene/7399/?report=expression. (B) Ush2a mRNA levels in the indicated mouse tissues. (C) Ush2a mRNA levels at the indicated developmental time periods in retinal tissue in mice. Whole embryo eyeballs were obtained at 12.5 days (12d) and 20.5 days (20d). d, days; w, weeks; m, months; nc, no DNA template; muscle, skeletal muscle; RPKM, reads per kilobase of transcript per million mapped reads. 
Table III. Expression of USH2A mRNA in human tissues.

\begin{tabular}{|c|c|c|c|}
\hline Tissue & $\begin{array}{c}\text { Number of } \\
\text { samples }\end{array}$ & $\begin{array}{l}\text { RPKM } \\
\text { values }\end{array}$ & Counts \\
\hline Adrenal & 3 & $0.163 \pm 0.040$ & 37,820 \\
\hline Appendix & 3 & $0.005 \pm 0.002$ & 984 \\
\hline Bone marrow & 4 & $0.001 \pm 0.001$ & 355 \\
\hline Brain & 3 & $0.017 \pm 0.008$ & 4,671 \\
\hline Colon & 5 & $0.005 \pm 0.004$ & 4,064 \\
\hline Duodenum & 2 & $0.015 \pm 0.001$ & 2,072 \\
\hline Endometrium & 3 & $0.005 \pm 0.0020$ & 1,114 \\
\hline Esophagus & 3 & $0.004 \pm 0.002$ & 1,592 \\
\hline Fat & 3 & $0.011 \pm 0.007$ & 2,513 \\
\hline Gall bladder & 3 & $0.004 \pm 0.002$ & 1,546 \\
\hline Heart & 4 & $0.06 \pm 0.0230$ & 30,633 \\
\hline Kidney & 4 & $0.076 \pm 0.068$ & 21,041 \\
\hline Liver & 3 & $1.691 \pm 0.096$ & 469,141 \\
\hline Lung & 5 & $0.006 \pm 0.004$ & 2,714 \\
\hline Lymph node & 5 & $0.003 \pm 0.004$ & 1,827 \\
\hline Ovary & 2 & $0.009 \pm 0.004$ & 2,706 \\
\hline Pancreas & 2 & $0.006 \pm 0.002$ & 1,498 \\
\hline Placenta & 4 & $0.005 \pm 0.005$ & 2,596 \\
\hline Prostate & 4 & $0.009 \pm 0.006$ & 2,782 \\
\hline Salivary gland & 3 & $0.015 \pm 0.009$ & 7,066 \\
\hline Skin & 3 & $0.006 \pm 0.003$ & 2,279 \\
\hline Small intestine & 4 & $0.007 \pm 0.004$ & 2,300 \\
\hline Spleen & 4 & $0.003 \pm 0.003$ & 1,410 \\
\hline Stomach & 3 & $0.009 \pm 0.003$ & 2,563 \\
\hline Testis & 7 & $0.341 \pm 0.222$ & 348,396 \\
\hline Thyroid & 4 & $0.003 \pm 0.002$ & 1,543 \\
\hline Urinary bladder & 2 & $0.006 \pm 0.004$ & 1,432 \\
\hline
\end{tabular}

RPKM, reads per kilobase of transcript per million mapped reads; USH2A, usherin.

searching the databases for Exome Aggregation Consortium (https://gnomad.broadinstitute.org/) and HGMD (http://www. hgmd.cf.ac.uk/ac/index.php) (Table II).

Sanger sequencing was performed to confirm the variants and analyze co-segregation (Fig. 3). The mutations (c.T449G, c.T10695A) in the USH2A gene were validated to be compound heterozygous in the proband (Fig. $3 \mathrm{C}$ and $\mathrm{H}$; pedigree II: 1), of which c.T449G was inherited from her father (Fig. 3A and F; pedigree I: 1) and c.T10695A was inherited from her mother (Fig. 3B and G; pedigree I: 2). The proband's son was revealed to be heterozygous c.T10695A with a normal phenotype (pedigree III: 1; Fig. 3D and I), and the proband's husband had a normal phenotype and with wild-type alleles (Fig. 3E and J; pedigree II: 2). Therefore, these mutations in the $U S H 2 A$ gene and the mutations were co-segregated with this clinical phenotype in this family. Both c.T449G and c.T10695A variants were absent in the blood samples of 200 normal ethnically matched controls. Taken together, this discovery highlighted co-segregation of the variants in this family and pinpointed their roles in the pathogenesis of Usher syndrome type IIA.
Functional effects of the variants c.T449G (p.L150*) and c.T10695A (p.Y3565*) in USH2A. By comparing of Homo sapiens (H. sapiens) USH2A protein to eight other species, including Pan troglodytes, Macaca mulatta, Canis lupus, Bos taurus, Rattus norvegicus, Mus musculus, Gallus gallus and Danio rerio, it was shown that USH2A is highly orthologously conserved (Fig. 4A). USH2A protein in H. sapiens contains a lamG-like jellyroll fold domain, laminin EGF domains, laminin-type EGF-like domains, laminin $\mathrm{G}$ domains, laminin $\mathrm{N}$-terminal (Domain VI) and many fibronectin type 3 domains (Fig. 4A and B). The c.T449G (p.L150*) variant is located in the lamG-like jellyroll fold domain (aa.146-aa.283), leading to the production of a truncated protein which had lost almost all the functional domains (Fig. 4B); whereas the c.T10695A (p.Y3565*) variant is located in one of the fibronectin type 3 domains (aa. 3503-aa. 3586), leading to the production of a truncated protein, that lost several fibronectin type 3 domains at the C-terminus of USH2A (Fig. 4B). Together, these results showed that the $U S H 2 A$ pathogenic compound heterozygous variants c.T449G (p.L150*) and c.T10695A (p.Y3565*) may have caused Usher syndrome type IIA disease.

mRNA expression profiles of USH $2 A$ and Ush $2 a$. USH $2 A$ expression analysis in humans indicated that USH $2 A$ mRNA expression was highest in the liver, with a reads per kilobase of transcript per million mapped reads value of 1.691 \pm 0.096 , followed by the testis, and was low or very low in other tissues (Fig. 5A). RNA-seq values in different tissues are presented in Table III. No human eye tissues and developmental retinal stage tissues were available. Thus, the Ush $2 a$ expression in mice was studied. Ush $2 a$ mRNA expression levels were highly expressed in the retina and other indicated tissues including the lens, sclera and cornea (Fig. 5B); whereas expression was not detected in the tissues, uterus, ovary, testis, breast, spleen, kidney, liver, brain, intestine, skeletal muscle, and blood. Ush $2 a$ was also highly expressed in the latter four different developmental retinal stages following birth (Fig. 5C). These results suggested that USH2A may serve a vital role in retinal/eye function based on the very high levels of Ush $2 a 2$ expression in the retinal tissue and its highly ubiquitous expression in other eye tissues.

\section{Discussion}

The association between the variations in Usher syndrome-causative genes and the resultant Usher syndrome diseases or phenotypes in patients are highly variable; the genotype/phenotype associations are also divergent $(1,3,4)$. Since Eudy et al (5) first identified three mutations in the USH2A gene in patients with Usher syndrome type IIA with RP and hearing loss, additional USH $2 A$ mutations have been shown to be associated with Usher syndrome type IIA (10-12,32-39). Patients with autosomal recessive RP (arRP; RP39; OMIM, 613809) without hearing loss were also identified to possess $U S H 2 A$ mutations, highlighting the complexity of genotype/phenotype associations in this disease (4). For example, Rivolta et al (40) identified USH $2 A$ mutations in patients with arRP without hearing loss. Zlotogora (41) reviewed examples and identified arRP patients without hearing loss who possessed mutations in 
the $U S H 2 A$ gene. Compound pathogenic mutations in the USH $2 A$ gene in Chinese RP families were also recently identified (42).

In the present study, using TGS, Sanger sequencing and co-segregation analysis, compound heterozygous pathogenic nonsense mutations, c.T449G (p.L150*) and c.T10695A (p.Y3565*), were identified in the USH2A gene in a Chinese pedigree. USH2A, very large G-protein coupled receptor 1 (VLGR1), USH1 and electroneutral sodium bicarbonate exchanger 1 (NBC3) are co-expressed in the synaptic terminals in both retinal photoreceptors and inner ear hair cells of mice and rats. The scaffold proteins harmonin, USH2A, VLGR1 and NBC3 interact with each other to assemble a multiprotein complex (43). USH2 and $\mathrm{NBC} 3$ proteins are interaction partners in a network in the retina and inner ear $(43,44)$. These p.L150* and p.Y3565* truncated USH2A mutations are hypothesized to affect complex formation (45), thereby elucidating the genetic roles of these USH $2 A$ mutant alleles in Usher syndrome type IIA.

The patient examined in the present study was first diagnosed as a nonsyndromic RP as she did not claim to suffer from hearing loss. Based on gene diagnostic results, pure tone audiometry testing was performed which presented binaural sensorineural deafness. Thus, USH2A mutations with Usher syndrome type IIA and an association of genotype/phenotype have been successfully linked in the patient of the studied family. Pater et al (46) recently reassigned the diagnosis of Usher syndrome by identifying novel $U S H 2 A$ splicing variants. To the best of our knowledge, the USH2A variants c.T449G (p.L150*), c.T10695A (p.Y3565*) are novel, thereby extending the spectrum of known mutations associated with this disease.

By comparing the of $H$. sapiens USH2A to eight other species, it was demonstrated that the USH2A protein is highly conserved. Ush $2 a$ mRNA expression levels in mice were demonstrated to only be highly expressed in the retina, lens, sclera and cornea from the eye tissues, consistent with a previous study (5), suggesting that USH2A serves vital roles in the functions of retina/eye. In addition to its high expression in the retina, USH2A in humans, rat and mice is also expressed in the cochlea $(5,47)$. USH2A was found to a likely component of the interstereocilia ankle which links the inner ear sensory cells (12). Taken together, the present study showed that the USH2A compound heterozygous variant, c.T449G (p.L150*) and c.T10695A (p.Y3565*), resulted in Usher syndrome type IIA.

As a rare disorder and the most common inherited form of combined visual and hearing impairment, up to 14 genes (MYO7A, CDH23, USHIC, PCDH15, USHIG and CIB2 for USH I, USH2A, ADGRVI and WHRN for USH II, CLRN1 and HARS for USH III, and PDZD7, CEP250 and C2 orf71 for either type I, II, or III of USH) are associated with Usher syndrome, with USH2A being the most prevalent worldwide $(2,48,49)$. With genetic diagnosis for Usher syndrome, repairing specific mutations using CRISPR/Cas9 editing system may now be possible. For example, Fuster-García et al (48) investigated gene editing to target the mutation in fibroblasts of a USH patient bearing the c.2299delG homozygous variation, highlighting the potential of the CRISPR editing system for the treatment of Usher syndrome.

In conclusion, the present study is the first to identify the novel compound heterozygous variants c.T449G (p.L150*) and c.T10695A (p.Y3565*) in the USH2A gene, which caused Usher syndrome type IIA in the examined patient. These mutations expand upon the library of known mutations associated with Usher syndrome. TGS provides a useful gene diagnostic approach (50). The present discovery may assist in understanding the molecular pathogenesis of RP and Usher syndrome type IIA, and in the development of strategies for the prevention, diagnosis, therapy and genetic counseling of patients with Usher syndrome type IIA. Additionally, the recruitment of more patients with Usher syndrome is an aim of future studies.

\section{Acknowledgements}

The authors would like to thank Professor Rui Chen at the Baylor College of Medicine for technical assistance and Ms. Shelly Fu at the University of Houston and Baylor College of Medicine for assistance and reading the manuscript.

\section{Funding}

The project was funded by the National Natural Science Foundation of China (grant nos. 31701087, 30371493 and 81672887), the Joint Research Foundation of Luzhou City and Southwest Medical University (grant no.2018LZXNYD-YL01), and The Translational Medicine Foundation of Southwest Medical University (grant nos. 00031476 and 00031477).

\section{Availability of data and materials}

The data generated using high-throughput sequencing were not submitted to any public databases due to the containing information that may compromise the proband's privacy but are available from the corresponding author on reasonable request.

\section{Authors' contributions}

JuF designed and conceptualized the study. JiF and JC performed DNA extraction, PCR amplification, sequencing and data analysis. HL, QZ and CD recruited the clinical patients and were in charge of the clinical assessments. JuF, MAK and JP analyzed the data, wrote and revised the manuscript. All authors read and approved the final manuscript.

\section{Ethics approval and consent to participate}

The present study was approved by the Ethics Committee of Southwest Medical University. The protocol and procedures employed for mice experiments were ethically reviewed and approved by the Ethics Committee of Southwest Medical University.

\section{Patient consent for publication}

Not applicable.

\section{Competing interests}

The authors declare that they have no competing interests. 


\section{References}

1. Wei C, Yang L, Cheng J, Imani S, Fu S, Lv H, Li Y, Chen R, Leung ELH and Fu J: A novel homozygous variant of GPR98 causes Usher syndrome type IIC in a consanguineous Chinese family by next generation sequencing. BMC Med Genet 19: 99, 2018.

2. Mathur P and Yang J: Usher syndrome: Hearing loss, retinal degeneration and associated abnormalities. Biochim Biophys Acta 1852: 406-420, 2015.

3. Fu J, Shen S, Cheng J, Lv H and Fu J: A case of Usher syndrome type IIA caused by a rare USH2A homozygous frameshift variant with maternal uniparental disomy (UPD) in a Chinese family. J Cell Mol Med 24: 7743-7750, 2020.

4. Zhu X, Li X, Tian W, Yang Y, Sun K, Li S and Zhu X: Identification of novel USH2A mutations in patients with autosomal recessive retinitis pigmentosa via targeted nextgeneration sequencing. Mol Med Rep 22: 193-200, 2020.

5. Eudy JD, Weston MD, Yao S, Hoover DM, Rehm HL, Ma-Edmonds M, Yan D, Ahmad I, Cheng JJ, Ayuso C, et al: Mutation of a gene encoding a protein with extracellular matrix motifs in Usher syndrome type IIa. Science 280: 1753-1757, 1998.

6. Besnard T, Vaché C, Baux D, Larrieu L, Abadie C, Blanchet C, Odent S, Blanchet P, Calvas P, Hamel C, et al: Non-USH2A mutations in USH2 patients. Hum Mutat 33: 504-510, 2012.

7. Ebermann I, Phillips JB, Liebau MC, Koenekoop RK, Schermer B, Lopez I, Schäfer E, Roux AF, Dafinger C, Bernd A, et al: PDZD7 is a modifier of retinal disease and a contributor to digenic Usher syndrome. J Clin Invest 120: 1812-1823, 2010.

8. Ebermann I, Scholl HP, Charbel Issa P, Becirovic E, Lamprecht J, Jurklies B, Millán JM, Aller E, Mitter D and Bolz H: A novel gene for Usher syndrome type 2: Mutations in the long isoform of whirlin are associated with retinitis pigmentosa and sensorineural hearing loss. Hum Genet 121: 203-211, 2007.

9. Hmani-Aifa M, Benzina Z, Zulfiqar F, Dhouib H, Shahzadi A, Ghorbel A, Rebaï A, Söderkvist P, Riazuddin S, Kimberling WJ and Ayadi H: Identification of two new mutations in the GPR98 and the PDE6B genes segregating in a Tunisian family. Eur J Hum Genet 17: 474-482, 2009.

10. van Wijk E, Pennings RJ, te Brinke H, Claassen A, Yntema HG, Hoefsloot LH, Cremers FPM, Cremers CWRJ and Kremer H: Identification of 51 novel exons of the Usher syndrome type $2 \mathrm{~A}$ (USH2A) gene that encode multiple conserved functional domains and that are mutated in patients with Usher syndrome type II. Am J Hum Genet 74: 738-744, 2004.

11. Weston MD, Eudy JD, Fujita S, Yao S, Usami S, Cremers C, Greenberg J, Ramesar R, Martini A, Moller C, et al: Genomic structure and identification of novel mutations in usherin, the gene responsible for Usher syndrome type IIa. Am J Hum Genet 66: 1199-1210, 2000.

12. Adato A, Lefèvre G, Delprat B, Michel V, Michalski N, Chardenoux S, Weil D, El-Amraoui A and Petit C: Usherin, the defective protein in Usher syndrome type IIA, is likely to be a component of interstereocilia ankle links in the inner ear sensory cells. Hum Mol Genet 14: 3921-3932, 2005.

13. Huang L, Mao Y, Yang J, Li Y, Li Y and Yang Z: Mutation screening of the USH2A gene in retinitis pigmentosa and USHER patients in a han Chinese population. Eye (Lond) 32: $1608-1614,2018$

14. Zhang L, Cheng J, Zhou Q, Khan MA, Fu J, Duan C, Sun S, Lv H and $\mathrm{Fu}$ J: Targeted next-generation sequencing identified novel compound heterozygous variants in the $\mathrm{CDH} 23$ gene causing Usher syndrome type ID in a Chinese patient. Front Genet 11: $422,2020$.

15. Huang J, Fu J, Fu S, Yang L, Nie K, Duan C, Cheng J, Li Y, $\mathrm{Lv} \mathrm{H}$, Chen R, et al: Diagnostic value of a combination of next-generation sequencing, chorioretinal imaging and metabolic analysis: Lessons from a consanguineous Chinese family with gyrate atrophy of the choroid and retina stemming from a novel OAT variant. Br J Ophthalmol 103: 428-435, 2019.

16. Fu J, Li L and Lu G: Relationship between microdeletion on Y chromosome and patients with idiopathic azoospermia and severe oligozoospermia in the Chinese. Chin Med J (Engl) 115: 72-75, 2002.

17. Cheng J, Fu J, Zhou Q, Xiang X, Wei C, Yang L, Fu S, Khan MA, $\mathrm{Lv} \mathrm{H}$ and $\mathrm{Fu} \mathrm{J}$ : A novel splicing mutation in the PRPH2 gene causes autosomal dominant retinitis pigmentosa in a Chinese pedigree. J Cell Mol Med 23: 3776-3780, 2019.
18. Wang F, Wang H, Tuan HF, Nguyen DH, Sun V, Keser V, Bowne SJ, Sullivan LS, Luo H, Zhao L, et al: Next generation sequencing-based molecular diagnosis of retinitis pigmentosa: Identification of a novel genotype-phenotype correlation and clinical refinements. Hum Genet 133: 331-345, 2014.

19. Fu Q, Xu M, Chen X, Sheng X, Yuan Z, Liu Y, Li H, Sun Z, $\mathrm{Li} \mathrm{H}$, Yang L, et al: CEP78 is mutated in a distinct type of Usher syndrome. J Med Genet 54: 190-195, 2017.

20. Zhang Q, Xu M, Verriotto JD, Li Y, Wang H, Gan L, Lam BL and Chen R: Next-generation sequencing-based molecular diagnosis of 35 Hispanic retinitis pigmentosa probands. Sci Rep 6: 32792, 2016.

21. Koenekoop RK, Wang H, Majewski J, Wang X, Lopez I, Ren H, Chen Y, Li Y, Fishman GA, Genead M, et al: Mutations in NMNAT1 cause Leber congenital amaurosis and identify a new disease pathway for retinal degeneration. Nat Genet 44: 1035-1039, 2012

22. Cheng J, Peng J, Fu J, Khan MA, Tan P, Wei C, Deng X, Chen H and $\mathrm{Fu}$ J: Identification of a novel germline BRCA2 variant in a Chinese breast cancer family. J Cell Mol Med 24: 1676-1683, 2020

23. Marchler-Bauer A, Bo Y, Han L, He J, Lanczycki CJ, Lu S, Chitsaz F, Derbyshire MK, Geer RC, Gonzales NR, et al: CDD/SPARCLE: Functional classification of proteins via subfamily domain architectures. Nucleic Acids Res 45: D200-D203, 2017.

24. Imani S, Ijaz I, Shasaltaneh MD, Fu S, Cheng J and Fu J: Molecular genetics characterization and homology modeling of the CHM gene mutation: A study on its association with choroideremia. Mutat Res 775: 39-50, 2018.

25. Imani S, Cheng J, Fu J, Mobasher-Jannat A, Wei C, Mohazzab-Torabi S, Jadidi K, Khosravi MH, Shasaltaneh MD, Yang L, et al: Novel splicing variant c. $208+2 \mathrm{~T}>\mathrm{C}$ in BBS5 segregates with bardet-biedl syndrome in an Iranian family by targeted exome sequencing. Biosci Rep 39: BSR20181544, 2019.

26. Wei C, Xiao T, Cheng J, Fu J, Zhou Q, Yang L, Lv H and Fu J. Novel compound heterozygous EYS variants may be associated with arRP in a large Chinese pedigree. Biosci Rep 40: BSR20193443, 2020.

27. Fu J, Zhou B, Zhang L, Balaji KS, Wei C, Liu X, Chen H, Peng $\mathrm{J}$ and $\mathrm{Fu} \mathrm{J}$ : Expressions and significances of the angiotensin-converting enzyme 2 gene, the receptor of SARS-CoV-2 for COVID-19. Mol Biol Rep 47: 4383-4392, 2020.

28. National Research Council (US) Committee for the Update of the Guide for the Care and Use of Laboratory Animals: Guide for the care and use of laboratory animals. 8th edition. National Academies Press (US), Washington, DC, 2011.

29. Fu J, Cheng J, Zhou Q, Wei C, Chen H, Lv H and Fu J: A novel missense variant c.G644A (p.G215E) of the RPGR gene in a Chinese family causes X-linked retinitis pigmentosa. Biosci Rep 39: BSR20192235, 2019.

30. Yang L, Fu J, Cheng J, Wei C, Zhou Q, Ijaz I, Lv H and Fu J: A novel variant of the FZD4 gene in a Chinese family causes autosomal dominant familial exudative vitreoretinopathy. Cell Physiol Biochem 51: 2445-2455, 2018.

31. Liu X, Cheng J, Mei Z, Wei C, Khan MA, Peng J and Fu J: SCAR marker for identification and discrimination of specific medicinal lycium chinense miller from lycium species from ramp-PCR RAPD fragments. 3 Biotech 10: 334, 2020.

32. Dreyer B, Tranebjaerg L, Rosenberg T, Weston MD, Kimberling WJ and Nilssen O: Identification of novel USH2A mutations: Implications for the structure of USH2A protein. Eur J Hum Genet 8: 500-506, 2000.

33. Aller E, Jaijo T, Beneyto M, Nájera C, Oltra S, Ayuso C, Baiget M, Carballo M, Antiñolo G, Valverde D, et al: Identification of 14 novel mutations in the long isoform of USH2A in Spanish patients with Usher syndrome type II. J Med Genet 43: e55, 2006.

34. Baux D, Larrieu L, Blanchet C, Hamel C, Salah SB, Vielle A, Gilbert-Dussardier B, Holder M, Calvas P, Philip N, et al: Molecular and in silico analyses of the full-length isoform of usherin identify new pathogenic alleles in Usher type II patients. Hum Mutat 28: 781-789, 2007.

35. Yan D, Ouyang X, Patterson DM, Du LL, Jacobson SG and Liu XZ: Mutation analysis in the long isoform of USH2A in American patients with Usher syndrome type II. J Hum Genet 54: 732-738, 2009.

36. Testa F, Melillo P, Bonnet C, Marcelli V, de Benedictis A, Colucci R, Gallo B, Kurtenbach A, Rossi S, Marciano E, et al: Clinical presentation and disease course of Usher syndrome because of mutations in Myo7a or Ush2a. Retina 37: 1581-1590, 2017. 
37. Garcia-Garcia G, Aparisi MJ, Jaijo T, Rodrigo R, Leon AM, Avila-Fernandez A, Blanco-Kelly F, Bernal S, Navarro R, Diaz-Llopis M, et al: Mutational screening of the USH2A gene in Spanish USH patients reveals 23 novel pathogenic mutations. Orphanet J Rare Dis 6: 65, 2011

38. Ng TK, Tang W, Cao Y, Chen S, Zheng Y, Xiao X and Chen $\mathrm{H}$ : Whole exome sequencing identifies novel USH2A mutations and confirms Usher syndrome 2 diagnosis in Chinese retinitis pigmentosa patients. Sci Rep 9: 5628, 2019.

39. He C, Liu X, Zhong Z and Chen J: Mutation screening of the USH2A gene reveals two novel pathogenic variants in Chinese patients causing simplex usher syndrome 2. BMC Ophthalmol 20: 70, 2020.

40. Rivolta C, Berson EL and Dryja TP: Paternal uniparental heterodisomy with partial isodisomy of chromosome 1 in a patient with retinitis pigmentosa without hearing loss and a missense mutation in the Usher syndrome type II gene USH2A. Arch Ophthalmol 120: 1566-1571, 2002.

41. Zlotogora J: Parents of children with autosomal recessive diseases are not always carriers of the respective mutant alleles. Hum Genet 114: 521-526, 2004.

42. Fu YC, Chen N, Qiu ZL, Liu L and Shen J: Compound pathogenic mutation in the USH2A gene in Chinese RP families detected by whole-exome sequencing. Mol Med Rep 18: 5016-5022, 2018.

43. Reiners J, van Wijk E, Märker T, Zimmermann U, Jürgens K, te Brinke H, Overlack N, Roepman R, Knipper M, Kremer H and Wolfrum U: Scaffold protein harmonin (USH1C) provides molecular links between Usher syndrome type 1 and type 2 . Hum Mol Genet 14: 3933-3943, 2005.

44. Zou J, Chen Q, Almishaal A, Mathur PD, Zheng T, Tian C, Zheng QY and Yang J: The roles of USH1 proteins and PDZ domain-containing USH proteins in USH2 complex integrity in cochlear hair cells. Hum Mol Genet 26: 624-636, 2017.
45. Sorusch N, Bauß K, Plutniok J, Samanta A, Knapp B, Nagel-Wolfrum $K$ and Wolfrum U: Characterization of the ternary Usher syndrome SANS/ush2a/whirlin protein complex. Hum Mol Genet 26: 1157-1172, 2017.

46. Pater JA, Green J, O'Rielly DD, Griffin A, Squires J, Burt T, Fernandez S, Fernandez B, Houston J, Zhou J, et al: Novel Usher syndrome pathogenic variants identified in cases with hearing and vision loss. BMC Med Genet 20: 68, 2019.

47. Huang D, Eudy JD, Uzvolgyi E, Davis JR, Talmadge CB, Pretto D, Weston MD, Lehman JE, Zhou M, Seemayer TA, et al: Identification of the mouse and rat orthologs of the gene mutated in Usher syndrome type IIA and the cellular source of USH2A mRNA in retina, a target tissue of the disease. Genomics 80: 195-203, 2002.

48. Fuster-García C, García-García G, González-Romero E, Jaijo T Sequedo MD, Ayuso C, Vázquez-Manrique RP, Millán JM and Aller E: USH2A gene editing Using the CRISPR system. Mol Ther Nucleic Acids 8: 529-541, 2017.

49. Fuster-García C, García-García G, Jaijo T, Fornés N, Ayuso C, Fernández-Burriel M, Sánchez-De la Morena A, Aller E and Millán JM: High-throughput sequencing for the molecular diagnosis of Usher syndrome reveals 42 novel mutations and consolidates CEP250 as Usher-like disease causative. Sci Rep 8: $17113,2018$.

50. Adams DR and Eng CM: Next-generation sequencing to diagnose suspected genetic disorders. N Engl J Med 379: 1353-1362, 2018.

(i) $(-)$ This work is licensed under a Creative Commons Attribution-NonCommercial-NoDerivatives 4.0 International (CC BY-NC-ND 4.0) License. 